\title{
Operating Characteristics of Underlay Cognitive Relay Networks
}

\author{
Ankit Kaushik, Ralph Tanbourgi, Friedrich Jondral \\ Communications Engineering Lab \\ Karlsruhe Institute of Technology (KIT) \\ $\{$ Ankit.Kaushik, Ralph.Tanbourgi, Friedrich.Jondral\} @ kit.edu
}

\begin{abstract}
Understanding the performance of cognitive relay networks (CRNs) is of great interest. Recently, stochastic geometry is being used to model and characterize the performance of CRNs. It is a known fact that sensing is an integral part of the CRN, however, in most cases it is not perfect. Moreover, the model inaccuracies caused by simplifications and/or approximations when deriving the analytical expressions for characterizing CRNs may distort their true performance. With no sensing in the system, we determine a lower performance bound (LPB) that can be used to judge the reliability of other systems that include sensing and model approximations. Based on the LPB, the operating characteristics (OC) for the CRN are obtained, which determine the joint performance of the primary and secondary system. Finally, OC are used to investigate the system performance under different scenarios.
\end{abstract}

\section{INTRODUCTION}

Cognitive radio technology aims to provide promising yet liable solutions to cope with the ever increasing demand for electromagnetic spectrum [1]. Under the cognitive radio umbrella, different paradigms such as overlay, underlay and interweave are conceptualized [2]. These paradigms define the criterion to perform secondary access to the spectrum. Among them, underlay systems have been extensively studied in the literature. Besides, stochastic geometry [3] has been widely accepted for modelling and analysis of wireless networks. Recently, the concept of stochastic geometry has been applied also to the performance analysis of underlay cognitive relay networks (CRNs).

\section{A. Related Work and Motivation}

Primarily for an underlay system, it is necessary to characterize the interference caused by other transmitters in the system namely, primary and secondary transmitters. In reality, CRNs encounter variations in the interference due to random node locations, node mobility and fading. Stochastic geometry captures these variations and provides a probabilistic model for studying interference statistics. The characterization of interference in the context of underlay system has been extensively studied by [4]-[9]. The modelling of primary and secondary nodes using stochastic geometry was depicted by Ghasemi et al. [4], where the primary and secondary nodes locations were distributed according to a homogeneous Poisson Point Process (PPP) [10]. Moreover, the authors illustrated the interaction between the primary and secondary systems, whereby secondary transmitters (STs) detect the primary receivers (PRs) by sensing the beacons sent by PRs over a control channel. Hence, STs terminate their transmission when located within a certain distance from a PR, termed as exclusion zone.

Kusaladharma et al. [5] extended the analysis with exclusion zone to obtain an expression for the moment generating function of the interference. The authors further investigated the effect of beacon misdetection by the STs in [6]. However, the model used in [5], [6] has following limitations. First, the interference analysis is restricted to PR only, that is the interference at secondary receiver (SR) is not considered. Second, the exclusion zones stimulate dependency between the node locations of the PRs and STs. Hence, the resulting process is no longer a PPP. This aspect is not considered in the model. These limitations were resolved by Lee et al. [7], who modelled the distribution of STs based on Poisson cluster processes. Concerning the area spectral efficiency of the secondary system, the concept of exclusion zone is rather inefficient, as it restricts the transmissions of those STs that are inside the exclusion zone, although interfering signals may be in a deep fade. Song et al. [9] overcame this problem by relaxing the idea of exclusion zone. In this particular case too, the authors assume STs follow a homogeneous PPP.

Sensing is included in the CRN to reduce interference in the system, however the beacon is detected with a certain level of false alarm and missdetection [11]. We believe that at physical level, apart from sensing there exists numerous cause of imperfections which are inherent to the communication systems, e.g. RF distortions. The scope of the paper is limited to the imperfections induced due to sensing only. In addition to that, sensing introduces dependency in the model, which is difficult to capture. Ignoring this dependency may distort the true performance of the system. Moreover, in most works, the performance of the $\mathrm{CRN}$ is restricted to the outage probability at the PRs only. Outage at SRs is either not considered or dealt separately for the system optimization. These approaches lack a general expression that captures the complete system dynamics, and hence, may lead to sub-optimal solutions.

In this work, we model a CRN where secondary system do not perform sensing. By doing so, we avoid dealing with sensing imperfection and dependency between STs and PRs node locations, thereby reducing model complexity. In this way, we are able to obtain exact closed-form expressions for the distribution function of the signal-to-interference ratio (SIR) at PR and SR. Without sensing, the secondary system sustains 


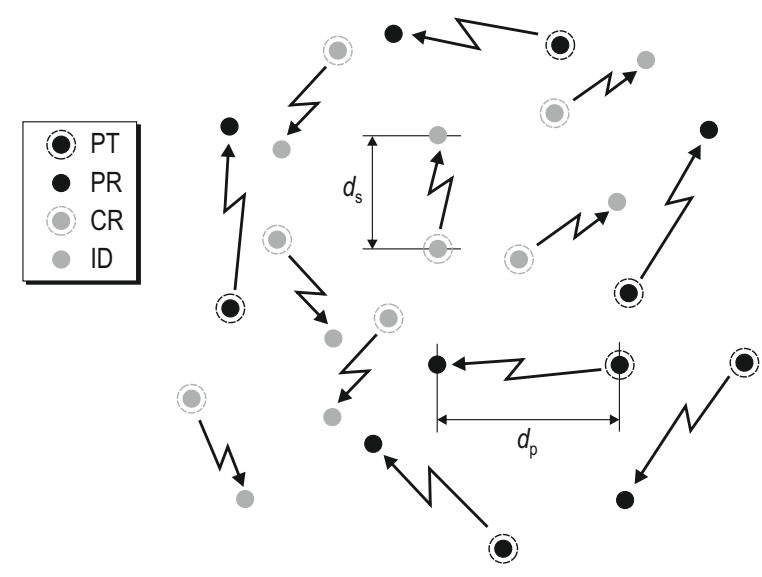

Fig. 1. A realization of a PPP for a cognitive relay network, depicting interference among the primary and secondary nodes.

the outage probability constraint at the PRs by regulating the transmit power at CRs for a given set of system parameters. Hence, we obtain an expression of the maximum transmit power at CRs.

Moreover, we consider outage probability constraints at the PR and SR jointly and derive operating characteristics (OC) for the considered CRN. The OC allows a joint characterization of outage probability constraint at the $\mathrm{PR}$ and the $\mathrm{SR}$, given a set of system parameters. The expression for the OC can be used to capture the performance of the entire CRN. The obtained expressions from our model can serve as a lower performance bound (LPB), that can be used to benchmark the performance of the other systems that consider sensing and contain model inaccuracies. From the system designer's perspective, the LPB can be an important tool for judging the system performance.

Finally, we employ the OC to the quantitatively analyze and compare the performance of the primary and secondary systems operating indoor, a scenario illustrated in [12], and outdoor. Our results indicate that systems operating indoor are more favorable than outdoor. Here indoor signifies that at least one of transmitter or receiver, corresponding to primary or secondary system, is operating indoor. For the outdoor case it is assumed that both the transmitter and receiver are operating outdoor.

\section{SySTEM MODEL}

Cognitive relay (CR) as introduced in [12], [13], is a network element that intends to fulfill the spectral requirements of the indoor devices (IDs). In this paper, we extend the concept of $\mathrm{CR}$ as underlay system to a CRN, that is, the secondary system deploys multiple CRs and examine their effect on the primary and secondary system. For downlink transmission from CR to ID, CR and ID correspond to ST and SR.

We assume the same transmit power for all CRs $P_{\mathrm{s}}$ and preclude any form of cooperation or coordination among them. In the model, we do not involve sensing at CRs, $P_{\mathrm{s}}$ can be regulated to sustain the constraint at the $\mathrm{PR}$. The regulation is necessary either at the system design or at time instants where the system parameters change. With its knowledge, $P_{\mathrm{s}}$ is used to characterize the capacity outage at SRs, which finally leads to a joint characterization of the $\mathrm{OC}$ for the $\mathrm{CRN}$ is determined.

\section{A. Network Model}

The locations of PTs are modelled by a stationary 2-D PPP $\Phi_{\mathrm{PT}}$ of density $\lambda_{\mathrm{p}}$. Similarly, the locations of CRs follow a stationary 2-D PPP $\Phi_{\mathrm{CR}}$ of density $\lambda_{\mathrm{s}}$, cf. Fig. 1 for an illustration. Due to the presence of transmitter-receiver pairs (PT-PR, CR-ID) in the system, the spatial distribution of PRs and IDs depends on $\Phi_{\mathrm{PT}}$ and $\Phi_{\mathrm{CR}}$. However, for the downlink PRs and IDs do not affect the system performance and hence do not need to be modelled.

All transmitted signals are subject to a distance-dependent path loss and small-scale channel fading. The distancedependent path loss is given as $\|\cdot\|^{-\alpha}$, where $\alpha>2$ is the path loss exponent. The small-scale channel fading is modelled as frequency-flat Rayleigh fading. Hence, the channel gains follow a unit-mean exponential distribution. The expressions presented in the paper are general and hence are applicable over a broad range of system parameters. However, to focus our analysis to the scenario described in [12], we consider a larger transmission distance and smaller node density for the primary system compared to the secondary system, i.e., $d_{\mathrm{p}} \geq d_{\mathrm{s}}$ and $\lambda_{\mathrm{p}} \leq \lambda_{\mathrm{s}}$, illustrated in Fig. 1. Lastly, for a simple distinction between different scenarios, we consider that both primary and secondary systems exists either in indoor or outdoor scenario. This corresponds to the same $\alpha$ for the primary and secondary systems. Moreover, it is considered that larger $\alpha$, e.g., $\alpha \geq 3$ and smaller $d_{\mathrm{p}}, d_{\mathrm{s}}$ are more likely to be designated to indoor [14].

Besides that, the primary and secondary system follow a time synchrnous slotted medium access with a certain channel access probability $\beta$. Applying the independent thinning property of the PPP, the set of simultaneous active PTs again forms a homogeneous PPP with density $\beta \lambda_{\mathrm{p}}[15]$. With no loss of generality, we consider the node density $\lambda_{\mathrm{p}}$ includes the channel access probability for the PT. Similarly for CRs, $\lambda_{\mathrm{s}}$ includes the channel access probability for the CR. Hence, according to this simplification, all PTs and CRs, with node densities $\lambda_{\mathrm{p}}$ and $\lambda_{\mathrm{s}}$, will transmit simultaneously.

\section{INTERFERENCE ANALYSIS}

In this section, we analyze the primary and secondary system jointly by deriving the OC. To obtain an expression for the OC, we first consider the SIR at a hypothetical PR, which we can place at origin $o$. Due to stationarity of $\Phi_{\mathrm{PT}}$ and $\Phi_{\mathrm{CR}}$, the SIR at PR is then

$$
\operatorname{SIR}_{\mathrm{PR}}=\frac{P_{\mathrm{p}} g_{o, \mathrm{p}} d_{\mathrm{p}}^{-\alpha}}{\sum_{i \in \Phi_{\mathrm{PT}}} P_{\mathrm{p}} g_{i}\left\|X_{i}\right\|^{-\alpha}+\sum_{j \in \Phi_{\mathrm{CR}}} P_{\mathrm{s}} g_{j}\left\|Y_{j}\right\|^{-\alpha}},
$$

where $P_{\mathrm{p}}$ and $P_{\mathrm{s}}$ are the transmit power at the PT and the CR. $\left\|X_{i}\right\|,\left\|Y_{i}\right\|$ are the distances of the $i^{\text {th }} \mathrm{PT}$ and $j^{\text {th }} \mathrm{CR}$ as interferers. $g_{o, \mathrm{p}}, g_{i}$ and $g_{j}$ are the channel gains for the 
intended PT, $i^{\text {th }} \mathrm{PT}$ and $j^{\text {th }} \mathrm{CR}$ as interferers. CRs must satisfy the outage constraint on $\mathrm{SIR}_{\mathrm{PR}}$, given by

$$
\mathbb{P}\left(\operatorname{SIR}_{\mathrm{PR}}<N_{\mathrm{p}}\right)=\mathrm{p}_{\text {out }, \mathrm{p}} \leq \epsilon_{\mathrm{p}},
$$

where $N_{\mathrm{p}}$ and $\epsilon_{\mathrm{p}}$ are the SIR threshold and outage probability constraint at the PR.

$\mathrm{SIR}_{\mathrm{PR}}$ depends on $\alpha$, primary system parameters $\lambda_{\mathrm{p}}, P_{\mathrm{p}}$ and $d_{\mathrm{p}}$, as well as on the secondary system parameters $\lambda_{\mathrm{s}}$ and $P_{\mathrm{s}}$. We assume that $\alpha$ and the primary system parameters are known at the system design, hence the additional interference at the PR from CRs can be regulated by choosing $P_{\mathrm{s}}$ and $\lambda_{\mathrm{s}}$ appropriately.

Before proceeding further, it is useful to characterize the performance of the system at the PR with only PTs as interferers.

Lemma 1: The success probability for a PR in absence of CRs is given by [15, (3.29)]

$$
\kappa=\mathbb{P}\left(\frac{P_{\mathrm{p}} g_{o, \mathrm{p}} d_{\mathrm{p}}^{-\alpha}}{\sum_{i \in \Phi_{\mathrm{PT}}} P_{\mathrm{p}} g_{i}\left\|X_{i}\right\|^{-\alpha}}>N_{\mathrm{p}}\right)=\exp \left(-\frac{2 \pi^{2} \lambda_{\mathrm{p}} c_{1} \frac{2}{\alpha}}{\alpha \sin \left(\frac{2 \pi}{\alpha}\right)}\right),
$$

where $c_{1}=N_{\mathrm{p}} d_{\mathrm{p}}^{\alpha}$.

Remark 1: $\kappa$ decreases exponentially with increase in $d_{\mathrm{p}}$, $\lambda_{\mathrm{p}}$ and $N_{\mathrm{p}}$. Moreover, $\alpha \sin (2 \pi / \alpha)$ is increasing and $N_{\mathrm{p}}^{2 / \alpha}$ is decreasing with $\alpha$, where $N_{\mathrm{p}}>1$, hence $\kappa$ increases with $\alpha$.

With the inclusion of CRs, the success probability $1-p_{\text {out,p }}$ and subsequently the constraint $1-\epsilon_{\mathrm{p}}$ at PR is limited by $\kappa$, hence $\kappa \geq 1-\epsilon_{\mathrm{p}}$. Therefore, it makes sense to account for the degradation in the primary system performance relative to $\kappa$.

Proposition 1: The relative degradation of the success probability at PR is given by

$$
\theta=\frac{1-\epsilon_{\mathrm{p}}}{\kappa},
$$

where $0 \leq \theta \leq 1$. For example, $\theta=0.95$ represents $5 \%$ degradation in success probability $\kappa$.

As discussed, all CRs transmit simultaneously with $P_{\mathrm{s}}$. For a certain node density $\lambda_{\mathrm{s}}$ and $\theta$, as per the system requirement it is important to determine the maximum transmit power $P_{\mathrm{s}}$ at CRs, such that the constraint in (2) is satisfied.

Lemma 2: For sustaining (2), the maximum transmit power $P_{\mathrm{s}}$ at $\mathrm{CRs}$ is

$$
P_{\mathrm{s}} \leq \frac{P_{\mathrm{p}}}{N_{\mathrm{p}}}\left(\frac{\alpha \sin \left(\frac{2 \pi}{\alpha}\right)}{2 \pi^{2} \lambda_{\mathrm{s}} d_{\mathrm{p}}^{2}} \ln \left(\frac{\kappa}{1-\epsilon_{\mathrm{p}}}\right)\right)^{\frac{\alpha}{2}} .
$$

Proof: To solve for $P_{\mathrm{s}}$, we consider the constraint in (2)

$$
\begin{aligned}
& \epsilon_{\mathrm{p}} \geq \mathbb{P}\left(\mathrm{SIR}_{\mathrm{PR}}<N_{\mathrm{p}}\right) \\
& \stackrel{(a)}{=} \mathbb{P}\left(g_{o, \mathrm{p}}<\frac{\sum_{i \in \Phi_{\mathrm{PT}}} N_{\mathrm{p}} g_{i}\left\|X_{i}\right\|^{-\alpha}+\sum_{j \in \Phi_{\mathrm{CR}}} \gamma N_{\mathrm{p}} g_{j}\left\|Y_{j}\right\|^{-\alpha}}{d_{\mathrm{p}}^{-\alpha}}\right) \\
& \stackrel{(b)}{=} 1-\mathbb{E}\left[\exp \left(-\frac{\sum_{i \in \Phi_{\mathrm{PT}}} N_{\mathrm{p}} g_{i}\left\|X_{i}\right\|^{-\alpha}+\sum_{j \in \Phi_{\mathrm{CR}}} \gamma N_{\mathrm{p}} g_{j}\left\|Y_{j}\right\|^{-\alpha}}{d_{\mathrm{p}}^{-\alpha}}\right)\right]
\end{aligned}
$$

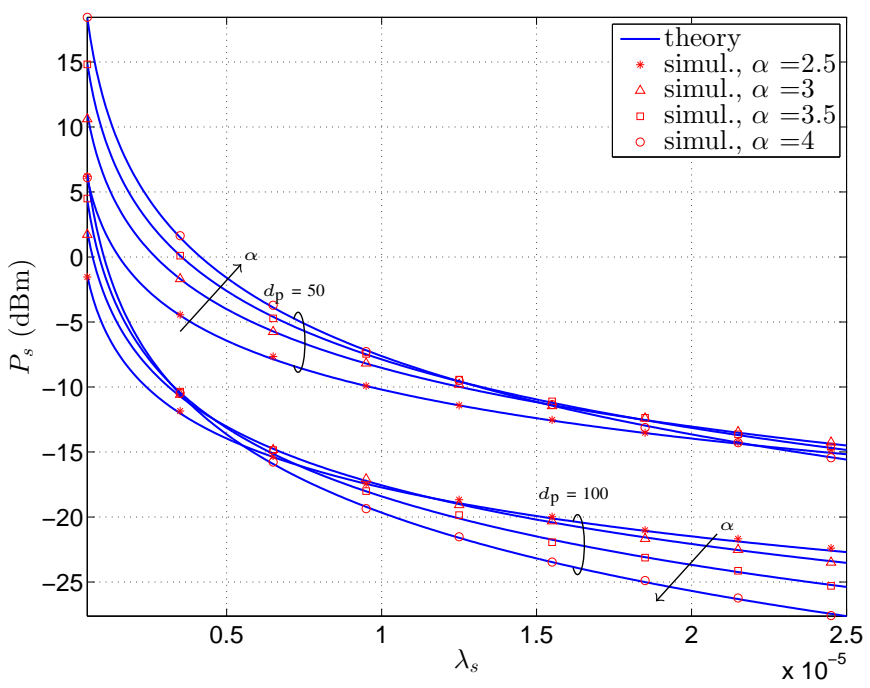

Fig. 2. Maximum transmit power of CRs for a given node density $\lambda_{\mathrm{s}}$, with different choices of $d_{\mathrm{p}}$ and $\alpha$, where $\theta=0.95, N_{\mathrm{p}}=10, P_{\mathrm{p}}=10 \mathrm{dBm}$ and $\lambda_{\mathrm{p}}=10^{-6}$ nodes $/ \mathrm{m}^{2}$.

$$
\begin{aligned}
& \stackrel{(c)}{=} 1-\mathbb{E}_{\Phi_{\mathrm{PT}}}\left[\mathbb{E}_{g}\left[\prod_{i \in \Phi_{\mathrm{PT}}} \exp \left(-\frac{N_{\mathrm{p}} g_{i}\left\|X_{i}\right\|^{-\alpha}}{d_{\mathrm{p}}^{-\alpha}}\right)\right]\right] \\
& \times \mathbb{E}_{\Phi_{\mathrm{CR}}}\left[\mathbb{E}_{g}\left[\prod_{j \in \Phi_{\mathrm{CR}}} \exp \left(-\frac{\gamma N_{\mathrm{p}} g_{j}\left\|Y_{j}\right\|^{-\alpha}}{d_{\mathrm{p}}^{-\alpha}}\right)\right]\right] \\
& \stackrel{(d)}{=} 1-\kappa \cdot \underbrace{\exp \left(-2 \pi^{2} \frac{c_{2}^{\frac{2}{\alpha}} \lambda_{\mathrm{s}}}{\alpha \sin \left(\frac{2 \pi}{\alpha}\right)}\right)}_{\theta}
\end{aligned}
$$

where $c_{2}=\gamma N_{\mathrm{p}} d_{\mathrm{p}}^{\alpha}$. (a) defines $\gamma=P_{\mathrm{s}} / P_{\mathrm{p}}$, (b) represents the expectation operation $\mathbb{E}[]$ over $\Phi_{\mathrm{PT}}, \Phi_{\mathrm{CR}}, g_{i}$ and $g_{j} .(c)$ follows from the independence between $\Phi_{\mathrm{PT}}$ and $\Phi_{\mathrm{CR}}$. $\mathbb{E}_{g}[]$ represents the expectation over channel gains for the nodes corresponding to either $\Phi_{\mathrm{PT}}$ or $\Phi_{\mathrm{CR}}$. Moreover, $(c)$ uses the probability generating functional (PGFL) [15. Appendix, A.5] and (3) to obtain $(d)$.

Remark 2: 6 represents the $\operatorname{SIR}_{\mathrm{PR}}$ outage probability $p_{\text {out, }}$. Similar to $\kappa$ in Remark 11, $\theta$ increases with $\alpha$ and decreases with $d_{\mathrm{p}}$. Hence, better performance, that is lower $p_{\text {out, } p}$ at PR is obtained for the scenarios with larger $\alpha$ and smaller $d_{\mathrm{p}}$. Now, $P_{\mathrm{s}}$ in Lemma 3 , obtained from our system model, can serve as a LPB for other systems with more sophisticated intereference coordination that involves sensing. From (5), it is clearly observed that $P_{\mathrm{s}}$ increases proportionally to $P_{\mathrm{p}}$, and inversely to $N_{\mathrm{p}}$. Moreover $\alpha / 2>1$, thus, for fixed $\alpha, P_{\mathrm{s}}$ decreases exponentially with $d_{\mathrm{p}}$ and $\lambda_{\mathrm{s}}$. It is worth noting that, $P_{\mathrm{s}}$ increases exponentially with $\ln \left(\kappa / 1-\epsilon_{\mathrm{p}}\right)=\ln (1 / \theta)$, cf. (47. Although $\alpha \sin (2 \pi / \alpha)$ is increasing with $\alpha$, still $P_{\mathrm{s}}$ is non-monotonic with $\alpha$, see Fig. 2. That is, for operation point 1 , where $\lambda_{\mathrm{s}}=2 \cdot 10^{-5}$ and $d_{\mathrm{p}}=100$, larger $\alpha$ degrades the signal power more than the interference from PTs and CRs. Hence to sustain a constant degradation $\theta=0.95$, CRs must operate at relatively lower $P_{\mathrm{s}}$ to satisfy (2), and vice versa for operation point 2 , where $\lambda_{\mathrm{s}}=1 \cdot 10^{-5}$ and $d_{\mathrm{p}}=50$. Hence, for a constant $\theta$, the effect of $\alpha$ is compensated by regulating $P_{\mathrm{S}}$ 
at CRs.

We now investigate the performance at the ID. The SIR at a hypothetical ID is

$$
\mathrm{SIR}_{\mathrm{ID}}=\frac{P_{\mathrm{s}} g_{o, \mathrm{~s}} d_{\mathrm{s}}^{-\alpha}}{\sum_{i \in \Phi_{\mathrm{PT}}} P_{\mathrm{p}} g_{i}\left\|X_{i}\right\|^{-\alpha}+\sum_{j \in \Phi_{\mathrm{CR}}} P_{\mathrm{s}} g_{j}\left\|Y_{j}\right\|^{-\alpha}} .
$$

Analogous to (1), due to stationarity of $\Phi_{\mathrm{PT}}$ and $\Phi_{\mathrm{CR}}$, the hypothetical ID is placed at $o . g_{o, \mathrm{~s}}$ represents the channel gain for the intended CR to ID at a distance $d_{\mathrm{s}}$. Moreover, from SIR $_{I D}$ the capacity at the ID is determined as

$$
\mathrm{C}_{\mathrm{ID}}=\log _{2}\left(1+\mathrm{SIR}_{\mathrm{ID}}\right)
$$

Hence, the performance of the secondary system can be determined from the SIR $_{I D}$ outage probability

$$
\mathbb{P}\left(\mathrm{SIR}_{\mathrm{ID}}<N_{\mathrm{s}}\right)=\mathrm{p}_{\text {out }, \mathrm{s}}
$$

or equivalently, from the $\mathrm{C}_{\mathrm{ID}}$ outage probability

$$
\mathbb{P}\left(\mathrm{C}_{\mathrm{ID}}<R_{\mathrm{s}}\right) \text {, }
$$

where $N_{\mathrm{s}}$ and $R_{\mathrm{s}}$ are the SIR and rate threshold at ID. Again, SIR ${ }_{\mathrm{ID}}$ depends on the system parameters $P_{\mathrm{p}}, \lambda_{\mathrm{p}}$ and $d_{\mathrm{p}}$ and $\alpha$, which are assumed to be known as well as on the parameters $\lambda_{\mathrm{s}}, d_{\mathrm{s}}$ and $P_{\mathrm{s}}$, which can be regulated by system designer.

Lemma 3: The $\mathrm{SIR}_{\mathrm{ID}}$ outage probability for ID in the CRN is given by

$$
p_{\text {out }, \mathrm{s}}=1-\left(1-\epsilon_{\mathrm{p}}\right)^{N_{\mathrm{s}}^{\frac{2}{\alpha}} m},
$$

where

$$
m=\frac{2 \pi^{2} \lambda_{\mathrm{s}} d_{\mathrm{s}}^{2}}{\alpha \sin \left(\frac{2 \pi}{\alpha}\right) \ln \left(\frac{\kappa}{1-\epsilon_{\mathrm{p}}}\right)} .
$$

Proof: The outage probability of $\mathrm{SIR}_{\mathrm{ID}}$ is evaluated as

$$
\begin{aligned}
p_{\text {out }, \mathrm{s}} & =\mathbb{P}\left(\mathrm{SIR}_{\mathrm{ID}}<N_{\mathrm{s}}\right) \\
& \stackrel{(a)}{=} 1-\exp \left(-2 \pi^{2} \frac{c_{3}^{\frac{2}{\alpha}} \lambda_{\mathrm{p}}}{\alpha \sin \left(\frac{2 \pi}{\alpha}\right)}\right) \exp \left(-2 \pi^{2} \frac{c_{4}^{\frac{2}{\alpha}} \lambda_{\mathrm{s}}}{\alpha \sin \left(\frac{2 \pi}{\alpha}\right)}\right) \\
& \stackrel{(b)}{=} 1-\kappa\left[\left(\frac{c_{3}}{c_{1}}\right)^{\frac{2}{\alpha}}\right] \cdot \theta\left[\left(\frac{c_{4}}{c_{2}}\right)^{\frac{2}{\alpha}}\right] \\
\quad \stackrel{(c)}{=} 1-(\kappa \cdot \theta) & {\left[\left(\frac{P_{\mathrm{p}} N_{\mathrm{s}} d_{\mathrm{s}}^{\alpha}}{P_{\mathrm{s}} N_{\mathrm{p}} d_{\mathrm{p}}^{\alpha}}\right)^{\frac{2}{\alpha}}\right] }
\end{aligned}
$$

where $c_{3}=(1 / \gamma) N_{\mathrm{s}} d_{\mathrm{s}}^{\alpha}$ and $c_{4}=N_{\mathrm{s}} d_{\mathrm{s}}^{\alpha}$ (a) follows the same procedure as in the proof of Lemma 2, (b) uses $e^{a b}=\left[e^{a}\right]^{b}$ and $(c)$ substitutes $P_{\mathrm{s}}$ from $(5)$ to obtain the final expression.

Substituting the expressions from (8), (9), (10) inside (11) in Lemma 3 to derive OC for the CRN.

Theorem 1: The operating characteristics for the CRN are given by

$$
\psi_{\mathrm{s}} \geq \mathbb{P}\left(\mathrm{C}_{\mathrm{ID}}<R_{\mathrm{s}}\right)=1-\left(1-\epsilon_{\mathrm{p}}\right)^{\left(2^{R_{\mathrm{s}}}-1\right)^{\frac{2}{\alpha}} m},
$$

where $\psi_{\mathrm{s}}$ is the outage probability constraint on the $\mathrm{C}_{\mathrm{ID}}$ outage probability given by 10 .

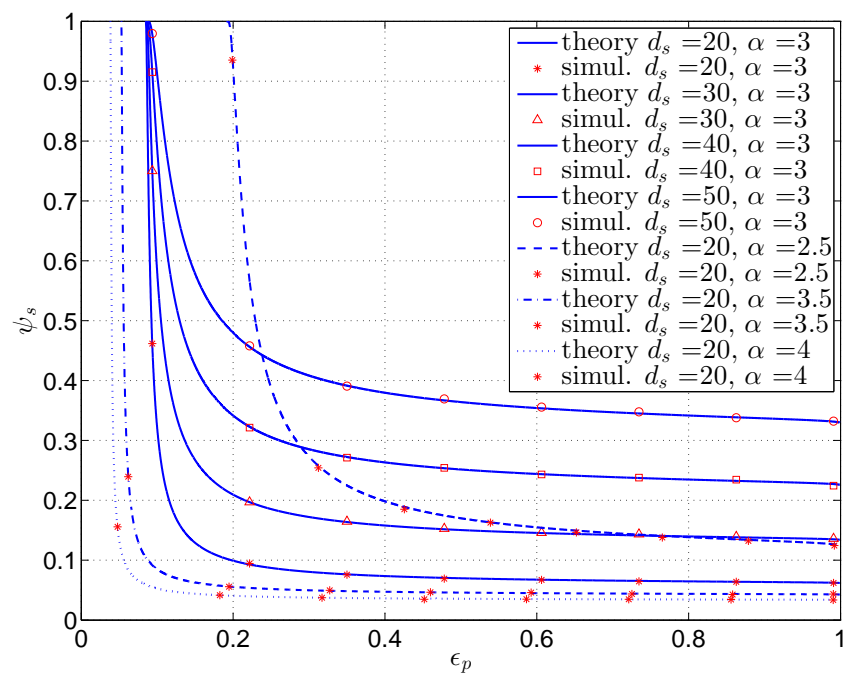

Fig. 3. OC illustrating $C_{\mathrm{ID}}$ outage $\psi_{\mathrm{s}}$ at the ID versus the $\operatorname{SIR} R_{\mathrm{PR}}$ outage $\epsilon_{\mathrm{p}}$ at the PR for the CRN with different choices of $d_{\mathrm{S}}$ and $\alpha$, where $N_{\mathrm{p}}=10$, $R_{\mathrm{S}}=2 \mathrm{bits} / \mathrm{sec} / \mathrm{Hz}, P_{\mathrm{p}}=10 \mathrm{dBm}, d_{\mathrm{p}}=50 \mathrm{~m}, \lambda_{\mathrm{p}}=10^{-6}$ nodes $/ \mathrm{m}^{2}$ and $\lambda_{\mathrm{s}}=10^{-5}$ nodes $/ \mathrm{m}^{2}$.

OC considers the $S_{R_{P R}}$ outage probability constraint at the $\operatorname{PR} \epsilon_{\mathrm{p}}$ and the $\mathrm{C}_{\mathrm{ID}}$ outage probability constraint at the ID $\psi_{\mathrm{s}}$.

Remark 3: Again, the expression (13) derived from our system model represents a LPB. Following Remark 11, $\epsilon_{\mathrm{p}}$ decreases with increase in $\alpha$ and decrease in $d_{\mathrm{p}}$, which results in a lower $\psi_{\mathrm{s}}$. Moreover, $m$ in the exponent of (13), decreases proportionally to $\lambda_{\mathrm{s}}$ and $d_{\mathrm{s}}^{2}$, which further lowers $\psi_{\mathrm{s}}$. Fixing $\theta$, the expression $\ln \left(\kappa / 1-\epsilon_{\mathrm{p}}\right)$ is held constant, however, $\alpha \sin (2 \pi / \alpha)$ increases and $\left(2^{R_{\mathrm{s}}-1}\right)^{\frac{2}{\alpha}}$ decrease with $\alpha$ for $R_{\mathrm{s}}>1$, hence, causes a decrease in $\psi_{\mathrm{s}}$. Thereby, the scenarios with larger $\alpha$, smaller $d_{\mathrm{p}}, d_{\mathrm{s}}$, e.g., $\alpha \geq 3, d_{\mathrm{p}} \approx 50 \mathrm{~m} d_{\mathrm{s}} \leq 30 \mathrm{~m}$, which attribute to indoor scenarios as illustrated in [12], result in a better OC, cf. Fig. 3 .

To extend the analysis of comparing different scenarios, we consider that the constraint at PR is fulfilled. The performance of the secondary system is investigated based on the first and second moments of $C_{I D}$. The variance $\operatorname{Var}\left[C_{I D}\right]$ may be useful indicator for judging quality of service (QoS) at the ID.

Corollary 1: The expected capacity at the ID is given by

$$
\mathbb{E}\left[\mathrm{C}_{\mathrm{ID}}\right]=\frac{1}{\ln 2} \int_{0}^{\infty} \frac{1}{1+x} e^{-\mu x^{\frac{2}{\alpha}}} \mathrm{d} x,
$$

where $\mu=-m \ln \left(1-\epsilon_{\mathrm{p}}\right)$ and $\mu \geq 0$. For the special case $\alpha=4$, the following closed-form expression for $\mathbb{E}\left[C_{I D}\right]$ exists

$$
\mathbb{E}\left[\mathrm{C}_{\mathrm{ID}}\right]=\frac{1}{\ln 2}\left[\sin (\mu)\left(\frac{\pi}{2}-\operatorname{si}(\mu)\right)-\cos (\mu) \operatorname{ci}(\mu)\right],
$$

where $\operatorname{si}(\cdot)$ and $\mathrm{ci}(\cdot)$ corresponds to sine and cosine integral [16].

Proof: See Appendix.

Corollary 2: The variance of capacity at the ID is given by

$\operatorname{Var}\left[\mathrm{C}_{\mathrm{ID}}\right]=\frac{2}{(\ln 2)^{2}} \int_{0}^{\infty} \frac{\ln (1+x)}{1+x} e^{-\mu x^{\frac{2}{\alpha}}} \mathrm{d} x-\mathbb{E}\left[\mathrm{C}_{\mathrm{ID}}\right]^{2}$. 


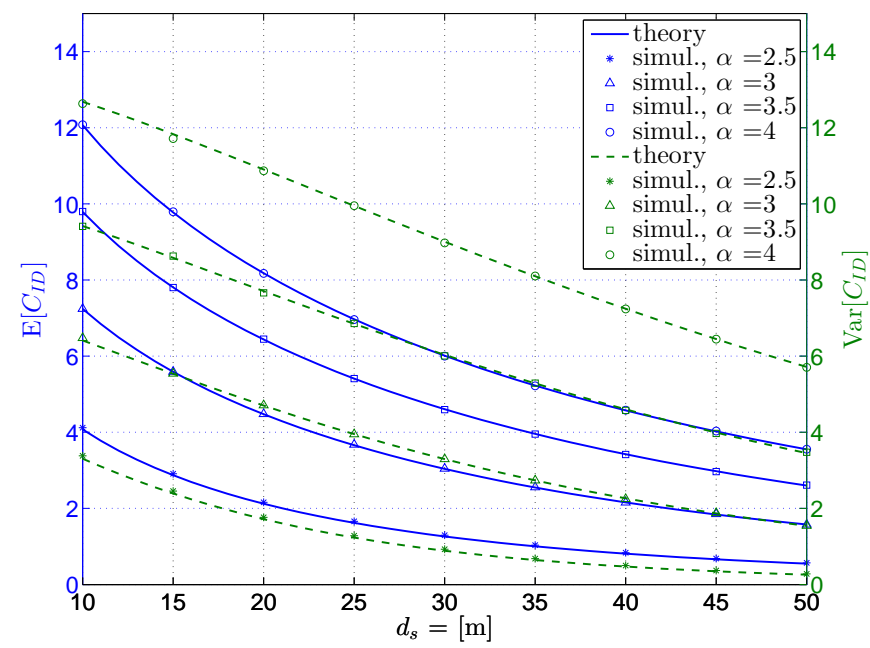

Fig. 4. $\mathbb{E}\left[C_{I D}\right]$ and $\operatorname{Var}\left[C_{I D}\right]$ of the capacity at the ID against the $d_{S}$, after the $\operatorname{SIR}_{\mathrm{PR}}$ constraint is satisfied, with different choices of $\alpha$, where $\theta=0.95, N_{\mathrm{p}}=10$ and $P_{\mathrm{p}}=10 \mathrm{dBm}, d_{\mathrm{p}}=50 \mathrm{~m}, \lambda_{\mathrm{p}}=10^{-6}$ nodes $/ \mathrm{m}^{2}$, $\lambda_{\mathrm{s}}=10^{-5}$ nodes $/ \mathrm{m}^{2}$.

Similar to $\mathbb{E}\left[C_{\mathrm{ID}}\right],(16)$ represents the analytical expression for $\operatorname{Var}\left[C_{I D}\right]$. For validation of the theoretical results, $\mathbb{E}\left[C_{I D}\right]$ and $\operatorname{Var}\left[\mathrm{C}_{\mathrm{ID}}\right]$ are computed numerically and compared with simulations.

$\mathbb{E}\left[\mathrm{C}_{\mathrm{ID}}\right]$ and $\operatorname{Var}\left[\mathrm{C}_{\mathrm{ID}}\right]$ depend on the area under $f(x) e^{-\mu x^{\frac{2}{\alpha}}}$, where $f(x)$ is $1 /(1+x)$ and $\ln (1+x) /(1+x)$, hence, $f(x)$ is independent of the system parameters. However, rate of decay for the exponential function is controlled by $\mu x^{\frac{2}{\alpha}}=-m \ln \left(1-\epsilon_{\mathrm{p}}\right) x^{\frac{2}{\alpha}}$. Considering $m$ and following the analysis similar to Remark 3 , large $\mathbb{E}\left[C_{I D}\right]$ is obtained for indoor scenarios, cf. Fig. 4 However, greater $\operatorname{Var}\left[\mathrm{C}_{\mathrm{ID}}\right]$ is observed for larger $\alpha$ and smaller $d_{\mathrm{s}}$, as larger $\alpha$ and smaller $d_{\mathrm{s}}$ scales the signal and larger $\alpha$ scales the interference from other PTs and CRs of SIRID, cf. (7). This may influence the QoS at ID.

\section{CONCLUSION}

The paper provides an extension to the concept of cognitive relay to cognitive relay network. Stochastic geometry is used to model the locations of the primary and secondary systems. The sensing imperfection and model inaccuracies for the cognitive ecosystem decreases the system's reliability. Motivated by this fact, we establish a lower performance bound to benchmark the performance of systems that include model inaccuracies and sensing. Furthermore, we obtain OC to jointly analyze the performance of primary and secondary systems. Based on the OC and the system parameters defined for an indoor scenario, it is indicated that the CRN operating indoor are propitious for the system.

\section{APPENDIX}

\section{EXPECTED CAPACITY AT ID}

Proof: Expected capacity for the ID is given as

$$
\mathbb{E}\left[\mathrm{C}_{\mathrm{ID}}\right]=\int_{0}^{\infty} R_{\mathrm{s}} \mathrm{d} F_{\mathrm{C}_{\mathrm{ID}}}\left(R_{\mathrm{s}}\right),
$$

where $F_{\mathrm{C}_{\mathrm{ID}}}\left(R_{\mathrm{S}}\right)$ is defined in 13 . In order to simplify 13 , $R_{\mathrm{S}}=x,\left(1-\epsilon_{\mathrm{p}}\right)=b, e=\frac{2}{\alpha}$ is substituted to give

$$
F_{\mathrm{C}_{\mathrm{ID}}}(x)=1-b^{\left(2^{x}-1\right)^{e} m}=1-c^{\left(2^{x}-1\right)^{e}},
$$

where $c=b^{m}$. Using (18) to evaluate (17) yields

$$
\mathbb{E}\left[C_{\mathrm{ID}}\right]=\ln 2 \cdot \ln c \int_{0}^{\infty} x e 2^{x}\left(2^{x}-1\right)^{e-1} c^{\left(2^{x}-1\right)^{e}} \mathrm{~d} x .
$$

Solving (19) obtains the general expression for the expected capacity 14, where $\ln (1 / c)=\mu$. For $\alpha=4,19$, gives

$$
\mathbb{E}\left[\mathrm{C}_{\mathrm{ID}}\right]=\frac{1}{\ln 2} \int_{0}^{\infty} \frac{2 x}{x^{2}+1} e^{-\mu x} \mathrm{~d} x .
$$

Applying [16, 3.354] to 20] yields [15].

\section{REFERENCES}

[1] S. Haykin, "Cognitive radio: brain-empowered wireless communications," IEEE J. Sel. Areas Commun., vol. 23, no. 2, pp. 201 - 220, Feb. 2005 .

[2] A. Goldsmith, S. Jafar, I. Maric, and S. Srinivasa, "Breaking Spectrum Gridlock With Cognitive Radios: An Information Theoretic Perspective," Proceedings of the IEEE, vol. 97, no. 5, pp. 894-914, May 2009.

[3] H. ElSawy, E. Hossain, and M. Haenggi, "Stochastic Geometry for Modeling, Analysis, and Design of Multi-Tier and Cognitive Cellular Wireless Networks: A Survey," IEEE Communications Surveys Tutorials, vol. 15 , no. 3, pp. 996-1019, Jun. 2013.

[4] A. Ghasemi and E. Sousa, "Interference Aggregation in SpectrumSensing Cognitive Wireless Networks," IEEE J. Sel. Topics Signal Process., vol. 2, no. 1, pp. 41-56, Feb. 2008.

[5] S. Kusaladharma and C. Tellambura, "Aggregate Interference Analysis for Underlay Cognitive Radio Networks," IEEE Wireless Commun. Lett., vol. 1, no. 6, pp. 641-644, Dec. 2012.

[6] — "Impact of Beacon Misdetection on Aggregate Interference for Hybrid Underlay-Interweave Networks," IEEE Commun. Lett., vol. 17, no. 11, pp. 2052-2055, Nov. 2013.

[7] C. Lee and M. Haenggi, "Interference and Outage in Poisson Cognitive Networks," IEEE Trans. Wireless Commun., vol. 11, no. 4, pp. 1392 1401, Apr. 2012.

[8] Y. Guo, Y. Ma, K. Niu, and J. Lin, "Transmission Capacity of Cognitive Radio Networks with Interference Avoidance," in Vehicular Technology Conference (VTC Spring), 2013 IEEE 77th, Jun. 2013, pp. 1-5.

[9] X. Song, C. Yin, D. Liu, and R. Zhang, "Spatial Throughput Characterization in Cognitive Radio Networks with Threshold-Based Opportunistic Spectrum Access," IEEE J. Sel. Areas Commun., vol. PP, no. 99, pp. 115, Nov. 2014.

[10] M. Haenggi, Stochastic Geometry for Wireless Networks. Cambridge University Press, 2013.

[11] R. Tanbourgi, J. Elsner, H. Jaekel, and F. K. Jondral, "Adaptive Frequency Hopping in Ad Hoc Networks with Rayleigh Fading and Imperfect Sensing," IEEE Wireless Commun Lett, vol. 1, no. 3, pp. 185188, Jun. 2012.

[12] A. Kaushik, M. Mueller, and F. K. Jondral, "Cognitive Relay: Detecting Spectrum Holes in a Dynamic Scenario," in Proceedings of the Tenth International Symposium on Wireless Communication Systems (ISWCS 2013), Apr. 2013, pp. 1-2.

[13] A. Kaushik, M. R. Raza, and F. K. Jondral, "On the Deployment of Cognitive Relay as Underlay Systems," in 9th International Conference on Cognitive Radio Oriented Wireless Networks and Communications, 2014. CROWNCOM '14., Jun. 2014.

[14] D. Tse and P. Viswanath, Fundamentals of Wireless Communication. Cambridge University Press, 2005.

[15] M. Haenggi and R. K. Ganti, "Interference in Large Wireless Networks," Foundations and Trends in Networking, vol. 3, no. 2, pp. 127-248, 2008, available at http://www.nd.edu/ mhaenggi/pubs/now.pdf

[16] I. S. Gradshteyn and I. M. Ryzhik, Table of Integrals, Series, and Products, 6th ed. San Diego, CA: Academic Press., 2000. 